\title{
NHERF1 and tumor microenvironment: a new scene in invasive breast carcinoma
}

\author{
Concetta Saponaro ${ }^{1}$, Alessandro Vagheggini', Emanuela Scarpi ${ }^{2}$, Matteo Centonze ${ }^{1}$, Ivana Catacchio', \\ Ondina Popescu ${ }^{3}$, Maria Irene Pastena ${ }^{3}$, Francesco Giotta ${ }^{4}$, Nicola Silvestris ${ }^{5+}$ and Anita Mangia ${ }^{1 *+}$
}

\begin{abstract}
Background: Tumor microenvironment (TME) includes many factors such as tumor associated inflammatory cells, vessels, and lymphocytes, as well as different signaling molecules and extracellular matrix components. These aspects can be de-regulated and consequently lead to a worsening of cancer progression. In recent years an association between the scaffolding protein $\mathrm{Na}^{+} / \mathrm{H}^{+}$exchanger regulatory factor 1 (NHERF1) and tumor microenvironment changes in breast cancer (BC) has been reported.

Methods: Subcellular NHERF1 localization, vascular endothelial growth factor (VEGF), its receptor VEGFR1, hypoxia inducible factor 1 alpha (HIF-1a), TWIST1 expression and microvessel density (MVD) in 183 invasive BCs were evaluated, using immunohistochemistry on tissue microarrays (TMA). Immunofluorescence was employed to explore protein interactions.

Results: Cytoplasmic NHERF1(CNHERF1) expression was directly related to cytoplasmic VEGF and VEGFR1 expression ( $p=0.001$ and $p=0.027$ respectively), and inversely to nuclear HIF-1a $(p=0.021)$ and TWIST1 $(p=0.001)$. Further, immunofluorescence revealed an involvement of tumor cells with NHERF1 positive staining in neo-vascular formation, suggesting a "mosaic" structure development of these neo-vessels. Survival analyses showed that loss of nuclear TWIST1 (nTWIST1) expression was related to a decrease of disease free survival (DFS) $(p<0.001$ ), while nTWIST1-/ mNHERF1+ presented an increased DFS with respect to nTWIST1+/mNHERF1- phenotype $(p<0.001)$. Subsequently, the analyses of nTWIST1+/CNHERF1+ phenotype selected a subgroup of patients with a worse DFS compared to nTWIST1-/CNHERF1- patients $(p=0.004)$.

Conclusion: Resulting data suggested a dynamic relation between NHERF1 and TME markers, and confirmed both the oncosuppressor role of membranous NHERF1 expression and the oncogene activity of cytoplasmic NHERF1.
\end{abstract}

Keywords: Tumor microenvironment, Tissue microarray, Immunohistochemical, NHERF1, Breast cancer

\section{Background}

Tumor microenvironment (TME) is a complex system in which different cell types, playing an important role in carcinogenesis and tumor-host relationship, co-exist. A bidirectional communication between cells and microenvironment has been highlighted in normal tissue homeostasis and also in the tissues involved in different diseases [1]. In this context the tumor generates and proliferates, surrounded by blood vessels, fibroblasts, inflammatory and immune cells, lymphocytes, different

\footnotetext{
* Correspondence: a.mangia@oncologico.bari.it

${ }^{\dagger}$ Equal contributors

${ }^{1}$ Functional Biomorphology Laboratory, IRCCS-Istituto Tumori "Giovanni Paolo II", 70124 Bari, Italy

Full list of author information is available at the end of the article
}

signaling molecules and extracellular matrix. Thus, the tumor can directly affect the microenvironment by the release of extracellular signals and immune escape mechanisms [2]. The interactions are often intricate and their characterization could permit to identify prognostic and predictive biomarkers useful for the development of new pharmacological targets and to provide novel therapeutical opportunities for cancer patients. This is also true for breast cancer (BC), one of the most diffused cancer in women, with a high incidence rate in all countries [3]. Recently, the heterogeneity of cellular and non-cellular components of TME has been underlined, including growth factors, cytokines, RNA, DNA, metabolites and matricellular proteins. They are involved in cancer onset 
and sustenance by supplying metabolites, energy, drug resistance environment, metastatic, growth and angiogenetic signals as well as evading immune surveillance $[4,5]$. Alterations and local changes in the concentration of angiogenic factors such as vascular endothelial growth factor (VEGF), its receptor (VEGFR1) and transcriptional targets of hypoxia inducible factor 1 alpha (HIF-1 $\alpha$ ), may trigger angiogenesis, by increasing microvessel density (MVD) in hypoxic breast TME [6, 7]. Among the factors distinguishing the TME, the epithelial-mesenchymal transition (EMT) plays an important role as well, and to date the involvement of some EMT markers has been extensively investigated [8]. TWIST1 is a basic helix-loop-helix transcription factor that contributes to carcinogenesis by triggering EMT, thereby influencing tumor invasion behavior [9]. TWIST1 over-expression was confirmed as having an essential role in tumor initiation, stemness, angiogenesis, invasion, metastasis, and chemo-resistance in a variety of carcinomas [10-13].

During recent years, our focus has been on the adaptor protein $\mathrm{Na}^{+} / \mathrm{H}^{+}$exchanger regulating factor 1 (NHERF1), a scaffold protein, with two PDZ and one EB (Ezrin-binding) domains, able to link different molecules and to drive several signal transduction pathways [14]. Its implication in different tumors such as predictive/ prognostic biomarkers has been extensively investigated [15-17], but little is known about NHERF1-TME relation. Our previous studies underlined its possible involvement in the orchestrate TME signaling pathway. In fact, its overexpression is implicated in the increase of invasive phenotype in breast cancer cells, in synergy with exposure to the hypoxic tumor microenvironment and with HIF-1 $\alpha$ expression [18]. Furthermore, at membranous and cytoplasmic level, its overexpression and colocalization with VEGFR1, a marker correlated with high metastasis risk and relapse has been evaluated [19]. A similar behavior has been also observed in advanced colorectal cancer (CRC), where overexpression of nuclear NHERF1 in association with hypoxic microenvironment and tumor invasive phenotype has been reported [20]. In a recent study, NHERF1 expression was positively correlated with VEGFR2 expression in CRC, suggesting NHERF1 involvement in disease progression by VEGFR2 signaling pathway [21]. The present study aimed to evaluate the possible involvement of NHERF1 and its association with the major TME markers as VEGF, VEGFR1, HIF-1 $\alpha$, MVD and TWIST1, in 183 invasive BC tumors. Moreover, we investigated possible specific immunophenotypes useful in identifying a subgroup of $\mathrm{BC}$ patients with a different clinical outcome, related to these TME markers. A better characterization of TME could allow the identification of new combinations of prognostic biomarkers valuable for clinical management and the development of novel therapeutic strategies.

\section{Methods}

\section{Patients and tissues}

The tissue microarray (TMA) utilized in this retrospective study included 549 samples of invasive BCs from 183 patients. The patients were subjected to primary surgery with nodal dissection at the Institute IRCCS Istituto Tumori "Giovanni. Paolo II" of Bari, Italy, in the years 2002-2003. For each tumor, 3 punches of cancerous tissues were available. The median age of patients was 49 years (range 24-83). For the subgroup of patients with follow-up $(64,5 \%)$, the median follow-up time was 82 months (range 1-162 months). TNM classification, tumor size, grade, perineoplastic invasion, estrogen receptor (ER), progesterone receptor (PgR), proliferative activity (MIB1) and HER2 status were provided by the Pathology Department of our Institute. The clinicopathological characteristics of the patients are listed in Table 1. Tumors with ER or PgR expression were classified as positive when nuclear staining was found in $>10 \%$. MIB1 nuclear staining was used to assess the proliferative activity, with a cut off value of $20 \%$ positive cells to indicate the tumors with MIB1 > $20 \%$ as highly proliferating. This cut off is the median value of scores relative to all breast tumors samples analysed during these years within our Institute. HER2 protein expression was studied using a monoclonal antibody (MoAb clone CB11; Novocastra Laboratories, Ltd., Newcastle, UK) and scored in accordance with the HercepTest scoring system (Food and Drug Administration): 0 indicated no membranous immunoreactivity or $<10 \%$ of cells reactive; $1+$, an incomplete membranous reactivity in $>10 \%$ of cells; $2+, \geq 10 \%$ of cells with weak to moderate complete membranous reactivity; and 3+, strong and complete membranous reactivity in $>10 \%$ of cells. Cytoplasmic immunoreactivity was ignored. Cases scoring 0 and $1+$ were classified as negative. HER2 was considered to be positive if immunostaining was $3+$ or if a score $2+$ showed gene amplification by fluorescence in situ hybridization (FISH). In FISH analyses, each copy of the HER2 gene and its centromere 17 (CEP17) reference was counted. The interpretation was in accordance with the criteria of $2007 \mathrm{ASCO} /$ CAP guidelines for HER2 testing in $\mathrm{BC}$, therefore positive if the HER2/CEP17 ratio was higher than 2,2 [22].

TMAs were constructed manually from formalin-fixed and paraffin-embedded tissues, according to standard procedures as previously described [15]. In brief, tumor target areas were selected from one donor block per patient, three $0.5 \mathrm{~mm}$ cores were punched out and transferred to the recipient TMA blocks. Each sample was arrayed in triplicate to minimize tissue loss and to overcome tumor heterogeneity, thus the three cores were representative of the whole specimen.

\section{Immunohistochemistry (IHC) and evaluation}

Four $\mu \mathrm{m}$-thick slices were cut from the TMA blocks and transferred to slides. The TMA slides were processed 
Table 1 Tumor characteristics of 183 invasive breast cancer patients

\begin{tabular}{|c|c|c|}
\hline Characteristics & $n$ & $(\%)$ \\
\hline \multicolumn{3}{|c|}{ Age: median value 49 (range 24-83) } \\
\hline$\leq 49$ years & 89 & $(48.6)$ \\
\hline$>49$ years & 94 & $(51.4)$ \\
\hline \multicolumn{3}{|l|}{ Histological type } \\
\hline IDC & 116 & $(90.7)$ \\
\hline ILC & 10 & $(5.5)$ \\
\hline Other & 7 & $(3.8)$ \\
\hline \multicolumn{3}{|l|}{ Histological grade } \\
\hline G1 & 29 & $(15.8)$ \\
\hline G2 & 84 & $(45.9)$ \\
\hline G3 & 70 & (38.3) \\
\hline \multicolumn{3}{|l|}{ Tumor size $(\mathrm{cm})$} \\
\hline$\leq 2 \mathrm{~cm}$ & 81 & $(44.8)$ \\
\hline$>2 \mathrm{~cm}$ & 100 & $(55.2)$ \\
\hline Unknown & 2 & \\
\hline \multicolumn{3}{|l|}{ Lymph node status } \\
\hline Negative & 71 & $(40.8)$ \\
\hline Positive & 103 & $(59.2)$ \\
\hline Unknown & 9 & \\
\hline \multicolumn{3}{|l|}{ Estrogen receptor } \\
\hline ER-negative ( $\leq 10 \%)$ & 53 & $(29.0)$ \\
\hline ER-positive (> 10\%) & 130 & $(71.0)$ \\
\hline Unknown & 1 & \\
\hline \multicolumn{3}{|l|}{ Progesterone receptor } \\
\hline PgR-negative ( $\leq 10 \%)$ & 76 & $(41.5)$ \\
\hline PgR-positive (> 10\%) & 107 & $(58.5)$ \\
\hline \multicolumn{3}{|l|}{ MIB1 } \\
\hline Negative $(\leq 20 \%)$ & 77 & $(42.1)$ \\
\hline Positive (> 20\%) & 106 & $(57.9)$ \\
\hline \multicolumn{3}{|l|}{ HER2/neu } \\
\hline Negative $(0,1+)$ & 78 & $(50.6)$ \\
\hline Positive (3+) & 76 & $(49.4)$ \\
\hline Unknown & 29 & \\
\hline Triple negative tumors & 19 & $(10.4)$ \\
\hline \multicolumn{3}{|l|}{ Treatment } \\
\hline $\mathrm{CT}$ & 27 & $(25.5)$ \\
\hline $\mathrm{HT}$ & 16 & (15.1) \\
\hline $\mathrm{CT}+\mathrm{HT}$ & 63 & (39.6) \\
\hline Unknown & 77 & \\
\hline
\end{tabular}

and stained by the indirect immunoperoxidase method [23]. In brief, TMA slides were deparaffinised and partially rehydrated through absolute ethanol and 95\% ethanol series. Antigen retrieval was performed by the 0 .
$01 \mathrm{M}$ citrate buffer $(\mathrm{pH} 6.0)$ at $98{ }^{\circ} \mathrm{C}$ in a water bath from a minimum of 20 to a maximum of $45 \mathrm{~min}$. The slides were then allowed to cool for $30 \mathrm{~min}$ and the endogenous peroxidase activity was blocked for $10 \mathrm{~min}$ with $3 \% \mathrm{H}_{2} \mathrm{O}_{2}$. The primary antibodies, diluted in PBS/ BSA $1 \%$, were incubated on the slides at $4{ }^{\circ} \mathrm{C}$ overnight in a moist chamber. For anti-VEGFR1, $1 \mathrm{~h}$ incubation at room temperature was required. A polymer-based IHC detection system was used as the amplification system (EnVision + System-HRP Labelled Polymer Anti-Rabbit or Anti-Mouse secondary antibody, Dako, Carpinteria, CA, USA) according to the manufacture's instruction. The bound antibody was visualized by incubating the sections in 3-amino-9-ethylcarbazole (AEC + Substrate Chromogen, Dako, Carpinteria, CA, USA) for $15 \mathrm{~min}$, except for antiCD34, which requires the use of 3,3'-diaminobenzidine (Liquid DAB + Substrate Chromogen System, Dako, Carpinteria, CA, USA) for 8-10 min. Cell nuclei were counterstained with Mayer's Haematoxylin (Bio-Optica, MI, Italy) and the slides were mounted with aqueous mounting medium (Faramount Aqueous Mounting Medium, Dako, Carpinteria, CA, USA). The different analysed biomarkers, dilution, source/clone, the staining localization of antibody and the cut off [median value or immunohistochemical score (HIS)] used to classify positive versus negative cases are shown in Additional file 1: Table S1.

All immunostained samples were scored by doubleblinded independent observers who had no patient outcome and clinicopathological data information, and the mean of the three readings for each patient was calculated. If one core was uninformative, lost or contained no tumor tissue, the overall score applied was that of the remaining cores. The results from two observers were identical in most cases, and discrepancies were resolved by re-examination and consensus.

NHERF1 immunostaining was predominantly cytoplasmic (cNHERF1), however in some cases an intense nuclear (nNHERF1) and membranous (mNHERF1) staining were also demonstrated. These were scored separately and their significance was evaluated. VEGF and VEGFR1 were mainly observed in the cytoplasm of breast cancer tissues (cVEGF and cVEGFR1), and the staining was evaluated as HIS or percentage of immunoreactive cells, respectively. Nuclear localization of HIF- $1 \alpha$ (nHIF-1 $\alpha)$ and TWIST1 (nTWIST1) was mainly observed. Cytoplasmic staining of HIF- $1 \alpha$ and TWIST1 was occasionally reported even if it was not evaluated for the aims of the study. The median value of immunoreactive cells was used as cut off for cNHERF1 ( $\geq 40 \%)$, nNHERF1 (>0\%), mNHERF1 (>0\%), nTWIST1 ( $\geq 4 \%)$, cVEGFR1 ( $>0 \%)$, nHIF-1 $\alpha(>0 \%)$ and MVD ( $\geq 15$ microvessels $/ \mathrm{mm}^{2}$ ). For cVEGF, the HIS was calculated by combining the quantity score (percentage of positive stained cells) with the staining intensity score [6]. The quantity score ranged from 0 to $4: 0=$ no 
immunoreactivity; $1 \leq 25 \%$ cells stained; $2=26-50 \%$ cells stained; $3=51-75 \%$ cells stained; and $4=\geq 76 \%$ cells stained. The staining intensity was scored as: 0 (negative), 1 (weak), 2 (moderate) and 3 (strong). Raw data were converted to IHS by adding the quantity score (0-4) to the staining intensity score (0-3). Theoretically, the scores can range from 0 to 7 . An IHS of 6-7 was considered a strong immunoreactivity; 3-5, moderate; 1-2, weak; and 0, negative. For our analyses, tumors presenting a moderate or strong score were cVEGF positive (IHS:3-7). Finally, microvessel counting was performed by identifying the areas which represented the highest vascular density, the so called "hot spots". The MVD measurements were made in the fields with a higher density of CD-34 positive cells and cell clusters at $200 \times$ magnification, as previously described [6]. Positive and negative controls were included in each staining run as indicated in the data sheet of each antibody. The accuracy, reliability, scoring strategy and reproducibility assessments of these antibodies have been validated in previous studies already published [6, 24-26].

\section{Immunofluorescence}

Immunofluorescent analysis was performed as described previously [18]. Briefly, formalin-fixed and paraffin embedded tissue serial sections of $3 \mu \mathrm{m}$ in thickness were deparaffinized with xylene, and rehydrated in an ethanol series. Antigen retrieval was carried out immersing slide in a $0.01 \mathrm{M}$ saline citrated buffer $(\mathrm{pH} 6.0)$ at $95{ }^{\circ} \mathrm{C}$ for $40 \mathrm{~min}$, then tissues were permeabilized with $0.1 \%$ Triton X100-Phosphate Buffered Saline for 15 min, blocked 30 min with 1\% Bovine Serum Albumin-Phosphate Buffered Saline and incubated overnight at $4{ }^{\circ} \mathrm{C}$ in a humidified chamber with a mouse monoclonal anti-CD34 (clone QBEND-10, dilution 1:20; Novocastra Lab. Ltd., UK) together with a rabbit polyclonal anti-NHERF1 (PA1-090, $1 \mu \mathrm{g} / 100 \mu \mathrm{L}$ dilution; Affinity Bio-Reagents). The slides were then incubated at room temperature for $1 \mathrm{~h}$ with the Alexa Fluor 488 and Alexa Fluor 568 immunoglobulin G secondary conjugated antibodies (1: 2000 dilution; Molecular Probes Inc., Eugene, OR, USA) and mounted with DAPI (ProLong ${ }^{\bullet}$ Gold antifade reagent; Molecular Probes Inc.). Positive control slides that were run simultaneously were used for assessing the quality of immunoreactivity. For negative controls, slide sections that were immunopositive were treated with $1 \%$ Bovine Serum Albumin instead of the primary antibody, and no reactivity was observed in any of these controls. Images were obtained on a BX40 microscope (Olympus, Tokyo, Japan) with a SenSys 1401E-Photometrics chargecoupled device camera. To verify protein colocalization, each acquired stack was merged by transforming the three channels corresponding to red (tetramethylrhodamine $\mathrm{B}$ isothiocyanate), green (fluorescein isothiocyanate) and blue (4',6-diamidino-2-phenylindole) into a single three-color stack by using the "RGB merge" command of Image software (National Institutes of Health Bethesda, MD).

\section{Follow up and statistical analysis}

Associations of tumor markers expression with various clinicopathological features and the among markers themselves were determined by the Chi-squared test for the independence of categorical variables. The presence of non-linear relationship between two continuous variables was assessed by the Kendall rank test.

The results from the immunohistochemical analyses of our biomarkers were assessed in relation to disease-free survival (DFS) and overall survival (OS). DFS (in months) was defined as the time from diagnosis to the date of loco-regional or distant recurrence, second invasive breast carcinoma, second primary cancer and/or death without evidence of breast cancer or to the date of last contact. OS (in months) was defined as the time from diagnosis to the date of last contact or of death from any cause. Univariate analyses of DFS and OS were carried out considering both tumor markers expression and clinicopathological variables: five-year DFS and OS and their 95\% confidence interval were based on Kaplan-Meier method, hazardratio (HR) were computed using the Cox proportional hazard regression model for DFS only (for OS it could not be computed due to the low number of deaths), and $p$-values of the log-rank test were used to infer on the equality of probability of an event (relapse for DFS or death for OS) in the two groups. Multivariate analyses on DFS (OS could not be analyzed due to the low number of deaths) were performed for tumor markers expressed as categorical and continuous variables using the Cox proportional hazard regression model; HRs were computed along with their p-values.

Data analysis was carried out using the statistical packages survival and survminer of the statistical language $R$, version 3.4 [27].

\section{Results \\ Clinicopathological characteristics}

The tumor characteristics of the 183 patients included in the study are listed in Table 1 . The $48.6 \%$ of patients were younger than 49 years. The majority had an invasive ductal carcinoma (IDC) (90.7\%), a moderate histological grade (G2) (45.9\%), and tumors larger than $2 \mathrm{~cm}$ (55.2\%) with axillary lymph node involvement (59.2\%). Furthermore, most patients had ER (71\%) and PgR (58. $5 \%)$ positive status, high MIB1 (57.9\%) and HER2/neu negative $(50.6 \%) ; 10.4 \%$ were triple negative breast cancers. The treatment of only 106 patients was known. Of these, $25.5 \%$ received adjuvant chemotherapy, $15.1 \%$ of ER-positive patients received hormonotherapy, and 39. $6 \%$ received adjuvant tamoxifen for 5 years after the end of chemotherapy. 


\section{Protein expression profiling}

The immunohistochemical expression of NHERF1, TWIST1, VEGFR1, VEGF, HIF-1 $\alpha$ and MVD, was evaluated on TMA sections. The frequency of expression of these biomarkers, in the whole cohort of tumor samples is reported in Additional file 2: Table S2. NHERF1 expression was detected in the apical membrane, cytoplasm, and nucleus of tumor samples. These different localizations were scored separately and their significance was evaluated. All breast cancers showed NHERF1 protein localized in the cytoplasm of tumor cells, of these $49 \%$ (76/154) overexpressed CNHERF1, according to the median value $\geq 40 \%$. Membranous NHERF1 and nNHERF1expression were detected in $13 \%(20 / 151)$ and $20 \%(30 / 149)$ of cases, respectively (median value $>0$ for both compartments). Cytoplasmic VEGF was expressed in $96 \%(144 / 150)$ of tumor cells, of these 54\% (81/151) overexpressed it, according to the IHS median value $\geq 3$. Cytoplasmic VEGFR1 and nuclear HIF1 $\alpha$ were expressed in about 50\% (80/161) and 37\% $(56 / 153)$ of tumor cells and, according to the median value $>0$ for both markers. Nuclear TWIST1 was expressed in $60 \%(84 / 140)$ of tumor cells, of these $51 \%(72 / 140)$ overexpressed it, according to the median value $\geq 4$. Tumors showed microvessel presence and 54\% (85/157) of them were characterized by a higher MVD, according to the median value $\geq 15$ microvessels $/ \mathrm{mm}^{2}$. Figure 1 shows an example of immunohistochemical staining pattern of NHERF1 and VEGF, VEGFR1, HIF-1 $\alpha$, TWIST1 and MVD expression in TMA tumor cores. NHERF1 was present in cytoplasmic compartment and less frequently at nuclear level, according with the median values of expression.
VEGF, VEGFR1, HIF-1 $\alpha$, TWIST1 and MVD expression was heterogeneous for intensity and expression patterns in the different tumor samples examined.

\section{Relationship between tumor markers and clinicopathological features}

A summary of significant association between tumor marker expressions and clinicopathological features are reported in Table 2. Among the BCs with cNHERF1 overexpression, $67.1 \%$ exhibited a significant association with tumor size $>2 \mathrm{~cm}(p=0.023)$ and $68.4 \%$ with MIB1 positive expression $(p=0.027)$. Further, negative cNHERF1 expression was related to PgR-positive expression $(p=0.023)$ and HER2/neu negative $(p=0.002)$ in $67.1 \%$ and $65.2 \%$ of cases respectively. The lack of nuclear NHERF1 was noticeably associated to tumor size $>2 \mathrm{~cm}(p=0.014)$, while its presence was linked to MIB1 negative expression $(p<0.001)$. Analyzing NHERF1 reactivity at membrane level, its absence was observed in IDCs $(\mathrm{p}=0.002)$ and positive lymph node status $(p=0.013)$; conversely mNHERF1 expression was significantly associated with tumor size $\leq 2 \mathrm{~cm}(p=0$. $039)$ and MIB1 negative $(p=0.019)$. A statistically significant relation was observed between mNHERF1 and PgRpositive expression $(p=0.034)$.

High cVEGF expression was present in $61.7 \%$ of younger patients $(p=0.006)$, and it showed a significant association with positive lymph node status $(p=0.010)$, higher tumor histological grade $(p=0.020)$, MIB1 positive expression $(p<0.001)$. CytoplasmicVEGF negative detection was related to HER2/neu negative expression $(p=0.006)$. Furthermore, a statistically significant relation was

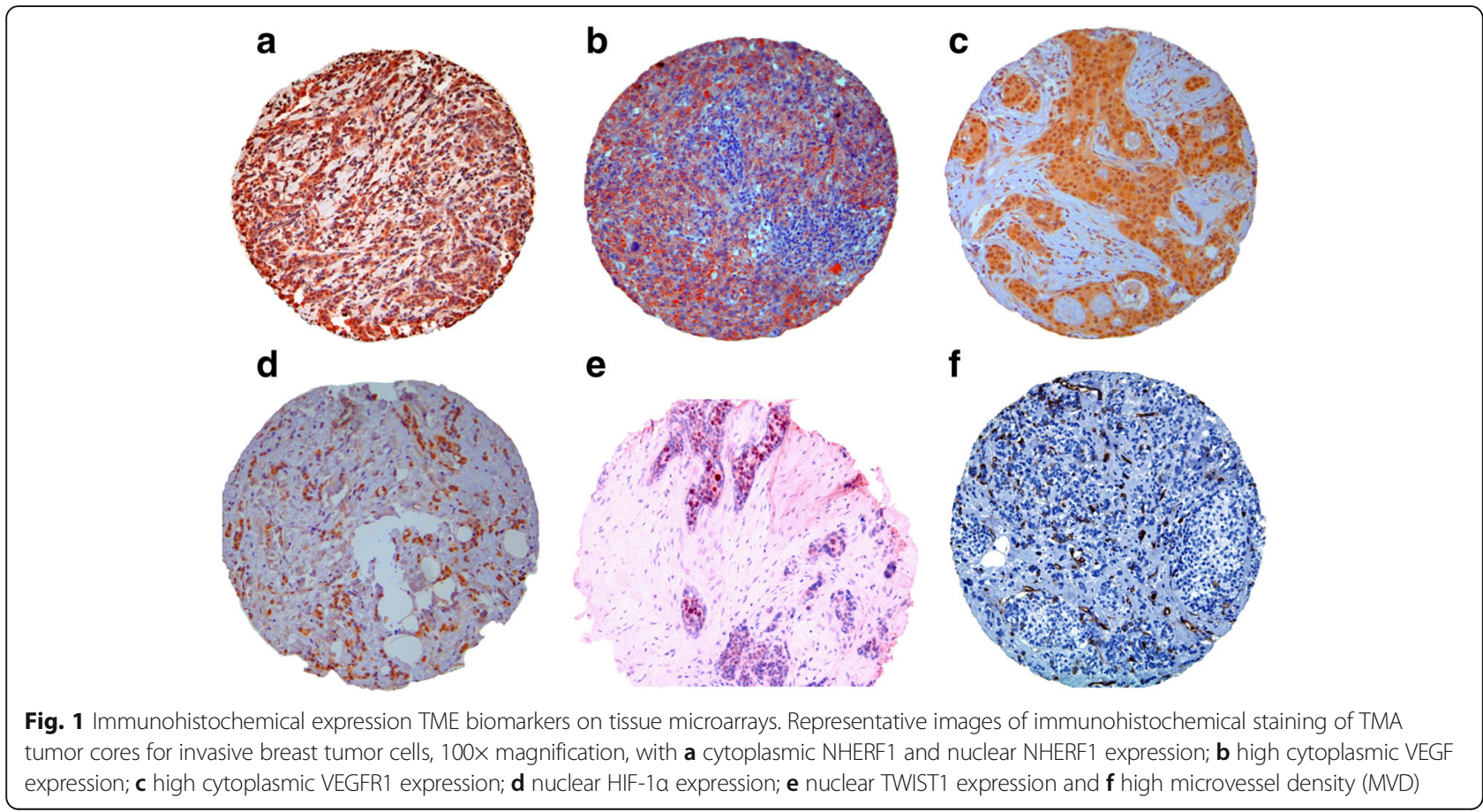


Table 2 Relationship between tumor markers and clinicopathological features

\begin{tabular}{|c|c|c|c|c|c|c|c|c|c|c|c|c|c|c|c|c|c|c|c|c|}
\hline \multirow[b]{3}{*}{ Characteristics } & \multicolumn{5}{|c|}{ CNHERF1 } & \multicolumn{5}{|c|}{ nNHERF1 } & \multicolumn{5}{|c|}{ mNHERF1 } & \multicolumn{5}{|c|}{ CVEGF } \\
\hline & \multicolumn{2}{|c|}{ Negative } & \multicolumn{2}{|c|}{ Positive } & \multirow[t]{2}{*}{$p$-value } & \multicolumn{2}{|c|}{ Negative } & \multicolumn{2}{|c|}{ Positive } & \multirow[t]{2}{*}{$p$-value } & \multicolumn{2}{|c|}{ Negative } & \multicolumn{2}{|c|}{ Positive } & \multirow[t]{2}{*}{$p$-value } & \multicolumn{2}{|c|}{ Negative } & \multicolumn{2}{|c|}{ Positive } & \multirow[t]{2}{*}{$p$-value } \\
\hline & $n$ & (\%) & $n$ & (\%) & & $n$ & (\%) & $n$ & (\%) & & $n$ & (\%) & $n$ & (\%) & & $n$ & (\%) & $n$ & (\%) & \\
\hline \multicolumn{21}{|l|}{ Age } \\
\hline$\leq 49$ years & 38 & $(52.1)$ & 40 & (52.6) & \multirow[t]{2}{*}{0.944} & 63 & $(52.9)$ & 15 & $(50.0)$ & \multirow[t]{2}{*}{0.773} & 70 & $(53.4)$ & 10 & $(50.0)$ & \multirow[t]{2}{*}{0.774} & 27 & (39.1) & 50 & $(61.7)$ & \multirow[t]{2}{*}{0.006} \\
\hline$>49$ years & 35 & $(47.9)$ & 36 & $(47.4)$ & & 56 & $(47.1)$ & 15 & $(50.0)$ & & 61 & $(46.6)$ & 10 & $(50.0)$ & & 42 & $(60.9)$ & 31 & (38.3) & \\
\hline \multicolumn{21}{|l|}{ Histological type } \\
\hline IDC & 66 & $(90.4)$ & 72 & (94.7) & \multirow[t]{3}{*}{0.511} & 111 & (93.3) & 27 & $(90.0)$ & \multirow[t]{3}{*}{0.820} & 125 & (95.4) & 15 & $(75.0)$ & \multirow[t]{3}{*}{0.002} & 59 & (85.5) & 78 & (96.3) & \multirow[t]{3}{*}{0.051} \\
\hline ILC & 3 & $(4.1)$ & 1 & $(1.3)$ & & 3 & $(2.5)$ & 1 & (3.3) & & 3 & $(2.3)$ & 1 & $(5.0)$ & & 4 & $(5.8)$ & 2 & $(2.5)$ & \\
\hline Other & 4 & $(5.5)$ & 3 & (3.9) & & 5 & $(4.2)$ & 2 & $(6.7)$ & & 3 & $(2.3)$ & 4 & (20.0) & & 6 & $(8.7)$ & 1 & $(1.2)$ & \\
\hline \multicolumn{21}{|l|}{ Tumor size $(\mathrm{cm})$} \\
\hline$\leq 2 \mathrm{~cm}$ & 37 & (51.4) & 25 & (32.9) & \multirow[t]{2}{*}{0.023} & 44 & $(37.0)$ & 18 & $(62.1)$ & 0.014 & 50 & $(38.2)$ & 12 & $(63.2)$ & 0.039 & 33 & $(47.8)$ & 28 & (35.0) & 0.112 \\
\hline$>2 \mathrm{~cm}$ & 35 & $(48.6)$ & 51 & $(67.1)$ & & 75 & $(63.0)$ & 11 & $(37.9)$ & & 81 & (61.8) & 7 & $(36.8)$ & & 36 & $(52.2)$ & 52 & $(65.0)$ & \\
\hline Lymph node sta & & & & & & & & & & & & & & & & & & & & \\
\hline Negative & 24 & (34.3) & 30 & $(41.7)$ & 0.365 & 42 & (36.8) & 12 & $(42.9)$ & 0.557 & 42 & (33.6) & 12 & $(63.2)$ & 0.013 & 33 & (51.6) & 24 & (30.4) & 0.010 \\
\hline Positive & 46 & $(65.7)$ & 42 & (58.3) & & 72 & (63.2) & 16 & (57.1) & & 83 & $(66.4)$ & 7 & (36.8) & & 33 & $(48.4)$ & 55 & (69.6) & \\
\hline Histological grac & & & & & & & & & & & & & & & & & & & & \\
\hline G1 & 15 & (20.5) & 11 & $(14.5)$ & 0.086 & 17 & (14.3) & 9 & $(30.0)$ & 0.077 & 20 & (15.3) & 6 & $(30.0)$ & 0.138 & 17 & (24.6) & 7 & (8.6) & 0.020 \\
\hline G2 & 38 & $(52.1)$ & 31 & $(40.8)$ & & 55 & $(46.2)$ & 14 & $(46.7)$ & & 60 & $(45.8)$ & 10 & $(50.0)$ & & 30 & $(43.5)$ & 37 & $(45.7)$ & \\
\hline G3 & 20 & (27.4) & 34 & $(44.7)$ & & 47 & (39.5) & 7 & (23.3) & & 51 & (38.9) & 4 & $(20.0)$ & & 22 & (31.9) & 37 & (45.7) & \\
\hline
\end{tabular}

Receptor status

\begin{tabular}{|c|c|c|c|c|c|c|c|c|c|c|c|c|c|c|c|c|c|c|c|c|}
\hline $\begin{array}{l}\text { ER-negative } \\
(\leq 10 \%)\end{array}$ & 20 & (27.4) & 23 & (30.3) & 0.700 & 38 & (31.9) & 5 & (16.7) & 0.099 & 39 & (29.8) & 4 & (20.0) & 0.367 & 13 & (18.8) & 32 & (39.5) & 0.006 \\
\hline $\begin{array}{l}\text { ER-positive } \\
(>10 \%)\end{array}$ & 53 & (72.6) & 53 & (69.7) & & 81 & $(68.1)$ & 25 & (83.3) & & 92 & $(70.2)$ & 16 & (80.0) & & 56 & $(81.2)$ & 49 & (60.5) & \\
\hline $\begin{array}{l}\text { PgR-negative } \\
(\leq 10 \%)\end{array}$ & 24 & (32.9) & 39 & (51.3) & 0.023 & 55 & $(46.2)$ & 8 & (26.7) & 0.053 & 59 & $(45.0)$ & 4 & (20.0) & 0.034 & 27 & (39.1) & 38 & $(46.9)$ & 0.338 \\
\hline $\begin{array}{l}\text { PgR-positive } \\
(>10 \%)\end{array}$ & 49 & $(67.1)$ & 37 & (48.7) & & 64 & (53.8) & 22 & (73.3) & & 72 & $(55.0)$ & 16 & (80.0) & & 42 & $(60.9)$ & 43 & (53.1) & \\
\hline \multicolumn{21}{|l|}{ MIB1 } \\
\hline $\begin{array}{l}\text { Negative } \\
(\leq 20 \%)\end{array}$ & 36 & (49.3) & 24 & (31.6) & 0.027 & 39 & (32.8) & 21 & (70.0) & $<0.001$ & 49 & (37.4) & 13 & (65.0) & 0.019 & 40 & $(58.0)$ & 23 & (28.4) & $<0.001$ \\
\hline $\begin{array}{l}\text { Positive } \\
(>20 \%)\end{array}$ & 37 & $(50.7)$ & 52 & (68.4) & & 80 & $(67.2)$ & 9 & (30.0) & & 82 & (62.6) & 7 & (35.0) & & 29 & $(42.0)$ & 58 & (71.6) & \\
\hline
\end{tabular}

HER2/neu 
Table 2 Relationship between tumor markers and clinicopathological features (Continued)

\begin{tabular}{|c|c|c|c|c|c|c|c|c|c|c|c|c|c|c|c|c|c|c|c|c|}
\hline \multicolumn{21}{|l|}{ Tumor size $(\mathrm{cm})$} \\
\hline$\leq 2 \mathrm{~cm}$ & 39 & $(48.8)$ & 29 & $(36.2)$ & 0.110 & 36 & $(50.0)$ & 31 & $(36.9)$ & 0.100 & 36 & $(37.1)$ & 29 & $(52.7)$ & 0.062 & 26 & $(38.8)$ & 32 & $(44.4)$ & 0.501 \\
\hline$>2 \mathrm{~cm}$ & 41 & $(51.2)$ & 51 & $(63.8)$ & & 36 & $(50.0)$ & 53 & (63.1) & & 61 & $(62.9)$ & 26 & $(47.3)$ & & 41 & $(61.2)$ & 40 & (55.6) & \\
\hline \multicolumn{21}{|l|}{ Lymph node status } \\
\hline Negative & 33 & $(44.0)$ & 27 & (35.1) & 0.260 & 26 & $(38.2)$ & 32 & (39.5) & 0.874 & 31 & $(34.4)$ & 26 & $(47.3)$ & 0.125 & 25 & (39.1) & 27 & (39.7) & 0.940 \\
\hline Positive & 42 & $(56.0)$ & 50 & $(64.9)$ & & 42 & $(61.8)$ & 49 & $(60.5)$ & & 59 & $(65.6)$ & 29 & $(52.7)$ & & 39 & $(60.9)$ & 41 & $(60.3)$ & \\
\hline \multicolumn{21}{|l|}{ Histological grade } \\
\hline G1 & 14 & $(17.3)$ & 11 & (13.8) & 0.246 & 12 & $(16.7)$ & 15 & (17.6) & 0.898 & 13 & $(13.4)$ & 12 & (21.4) & 0.001 & 10 & $(14.7)$ & 14 & (19.4) & 0.251 \\
\hline G2 & 40 & (49.4) & 32 & $(40.0)$ & & 32 & $(44.4)$ & 40 & $(47.1)$ & & 34 & (35.1) & 32 & (57.1) & & 26 & $(38.2)$ & 34 & $(47.2)$ & \\
\hline G3 & 27 & (33.3) & 37 & $(46.2)$ & & 28 & $(38.9)$ & 30 & (35.3) & & 50 & (51.5) & 12 & (21.4) & & 32 & $(47.1)$ & 24 & (33.3) & \\
\hline \multicolumn{21}{|l|}{ Receptor status } \\
\hline $\begin{array}{l}\text { ER-negative } \\
(\leq 10 \%)\end{array}$ & 21 & (25.9) & 29 & $(36.2)$ & 0.157 & 19 & $(26.4)$ & 26 & (30.6) & 0.562 & 32 & (33.0) & 16 & (28.6) & 0.570 & 24 & (35.3) & 15 & (20.8) & 0.056 \\
\hline $\begin{array}{l}\text { ER-positive } \\
(>10 \%)\end{array}$ & 60 & $(74.1)$ & 51 & (63.8) & & 53 & (73.6) & 59 & (69.4) & & 65 & $(67.0)$ & 40 & (71.4) & & 44 & (64.7) & 57 & (79.2) & \\
\hline $\begin{array}{l}\text { PgR-negative } \\
(\leq 10 \%)\end{array}$ & 26 & (32.1) & 42 & $(52.3)$ & 0.009 & 32 & $(44.4)$ & 33 & (38.8) & 0.476 & 48 & $(49.5)$ & 18 & (32.1) & 0.037 & 34 & $(50.0)$ & 30 & (41.7) & 0.323 \\
\hline $\begin{array}{l}\text { PgR-positive } \\
(>10 \%)\end{array}$ & 55 & (67.9) & 38 & $(47.5)$ & & 40 & (55.6) & 52 & $(61.2)$ & & 49 & $(50.5)$ & 38 & (67.9) & & 34 & $(50.0)$ & 42 & (58.3) & \\
\hline \multicolumn{21}{|l|}{ MIB1 } \\
\hline $\begin{array}{l}\text { Negative } \\
(\leq 20 \%)\end{array}$ & 37 & (45.7) & 29 & (36.2) & 0.224 & 32 & $(44.0)$ & 34 & $(40.0)$ & 0.574 & 31 & (32.0) & 29 & (51.8) & 0.016 & 22 & (32.4) & 38 & (52.8) & 0.015 \\
\hline $\begin{array}{l}\text { Positive } \\
(>20 \%)\end{array}$ & 44 & (54.3) & 51 & (63.8) & & 40 & (55.6) & 51 & $(60.0)$ & & 66 & $(68.0)$ & 27 & $(48.2)$ & & 46 & (67.6) & 34 & $(47.2)$ & \\
\hline \multicolumn{21}{|l|}{ HER2/neu } \\
\hline Negative $(0,1+)$ & 41 & (58.6) & 34 & $(44.7)$ & 0.095 & 34 & $(54.0)$ & 36 & $(48.0)$ & 0.485 & 40 & $(44.9)$ & 32 & (61.5) & 0.057 & 29 & $(46.8)$ & 35 & $(50.7)$ & 0.652 \\
\hline Positive (3+) & 29 & $(41.4)$ & 7 & (55.3) & & 29 & $(46.0)$ & 39 & $(52.0)$ & & 49 & $(55.1)$ & 20 & (38.5) & & 33 & $(53.2)$ & 34 & (49.3) & \\
\hline
\end{tabular}

$p$-value of Chi-squared test for the independence of categorical variables. Bold values indicate significance

observed between cVEGF negative and ER-positive expression $(p=0.006)$. Expression of cVEGFR1 was statistically prevalent in invasive tumor phenotype $(p=0.041)$ and PgR-positive expression $(p=0.009)$. G2 tumors presented high nHIF- $1 \alpha$ expression $(p=0.001)$, and this overexpression was directly associated with PgR-positive expression $(p=0.037)$ while nHIF- $1 \alpha$ negative expression was associated to MIB1 positive expression $(p=$ 0.016). Low nTWIST1 expression was statistically prevalent in high MIB1 tumors $(p=0.015)$. There were no statistically significant associations between MVD and the clinicopathological features.

\section{Association between protein expressions analyzed}

As regards the dichotomized variables, Chi-squared test for protein associations is summarized in Table 3. For the continuous variables, Kendall's $\tau$ for rank-based correlations between markers and relative heatmap are shown in Fig. 2a and b.

Of 183 total tumors, 138 could be assessed for both cVEGF and cNHERF1. In particular, among these 79 tumors over-expressed cNHERF1 and $65.8 \%$ had high
cVEGF expression as well; additionally, of 59 cases with negative cNHERF1, 66.1\% had negative cVEGF expression $(p<0.001)$. Cytoplasmic VEGF negative expression was also related to nuclear $(p=0.001)$ and membranous NHERF1 expression $(p=0.001)$ in $91.1 \%$ and $94.4 \%$ of cases, respectively. Kendall's $\tau$ for rank-based correlation underlined direct relationship between cVEGF and cNHERF1 $(\tau=0.323, p<0.001)$ and inverse between nNHERF1 $(\tau=-0.247, p<0.001)$ and mNHERF1 $(\tau=-0$. $178, p=0.012)$. One hundred and forty-seven tumors resulted evaluable for both cVEGFR1 and cNHERF1 expression. In particular, $53.7 \%$ the tumors showed cNHERF1 over-expression and 59.5\% had also high cVEGFR1 expression $(p=0.027)$. No statistically significant correlation was observed between cVEGFR1 and nuclear and membranous NHERF1 expression. Kendall's $\tau$ test for continuous data showed an inverse association between cVEGFR1 and MNHERF1 ( $\mathrm{\tau}=-0.161, p=0.024)$, while confirming the direct association between cVEGFR1 and cNHERF1 expression ( $\tau=0.189, p=0.003)$. Nuclear HIF- $1 \alpha$ and cNHERF1 expression was observed in 142 tumors. Low nHIF- $1 \alpha$ expression was related to $60.4 \%$ of the cNHERF1 


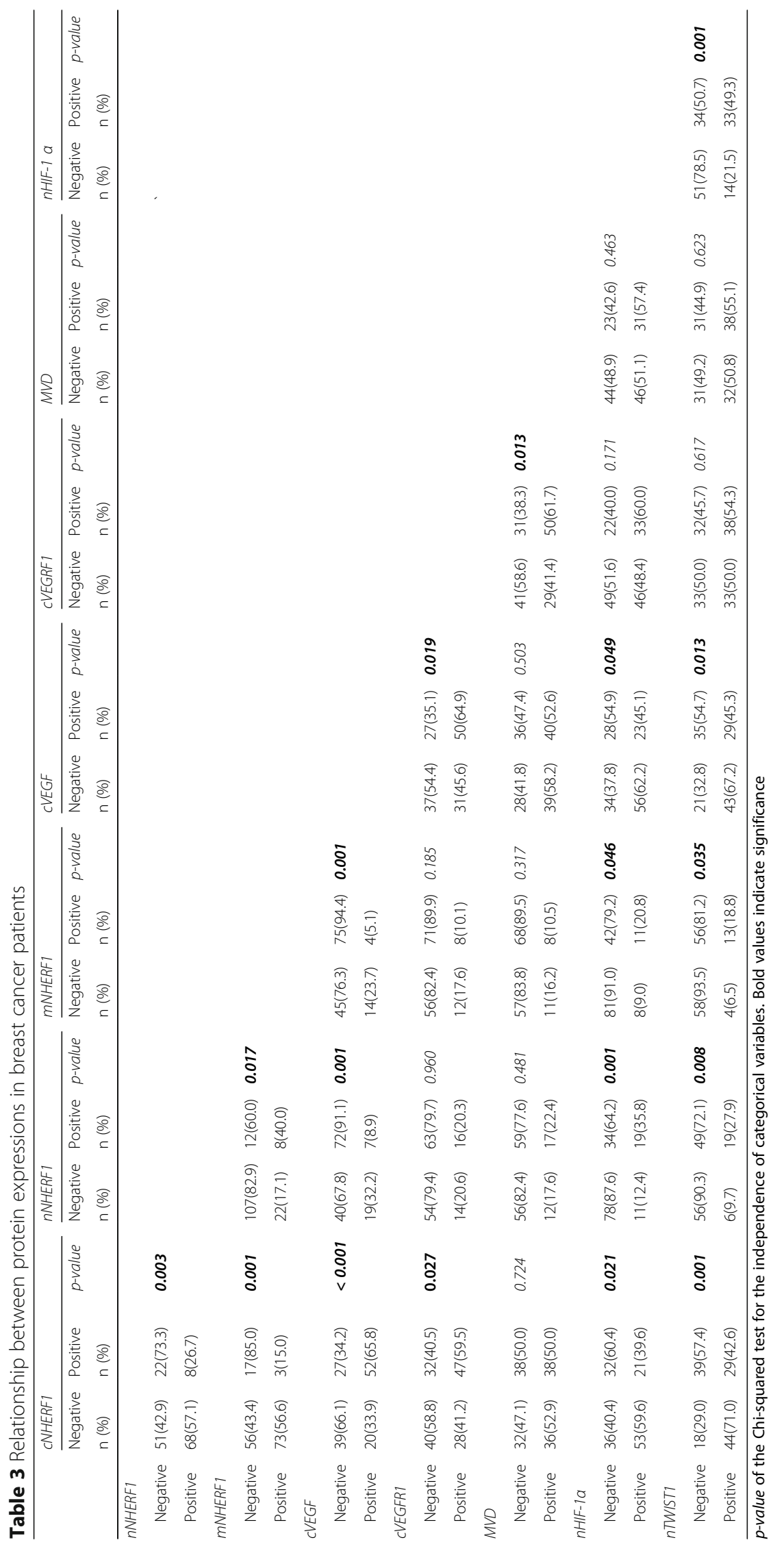




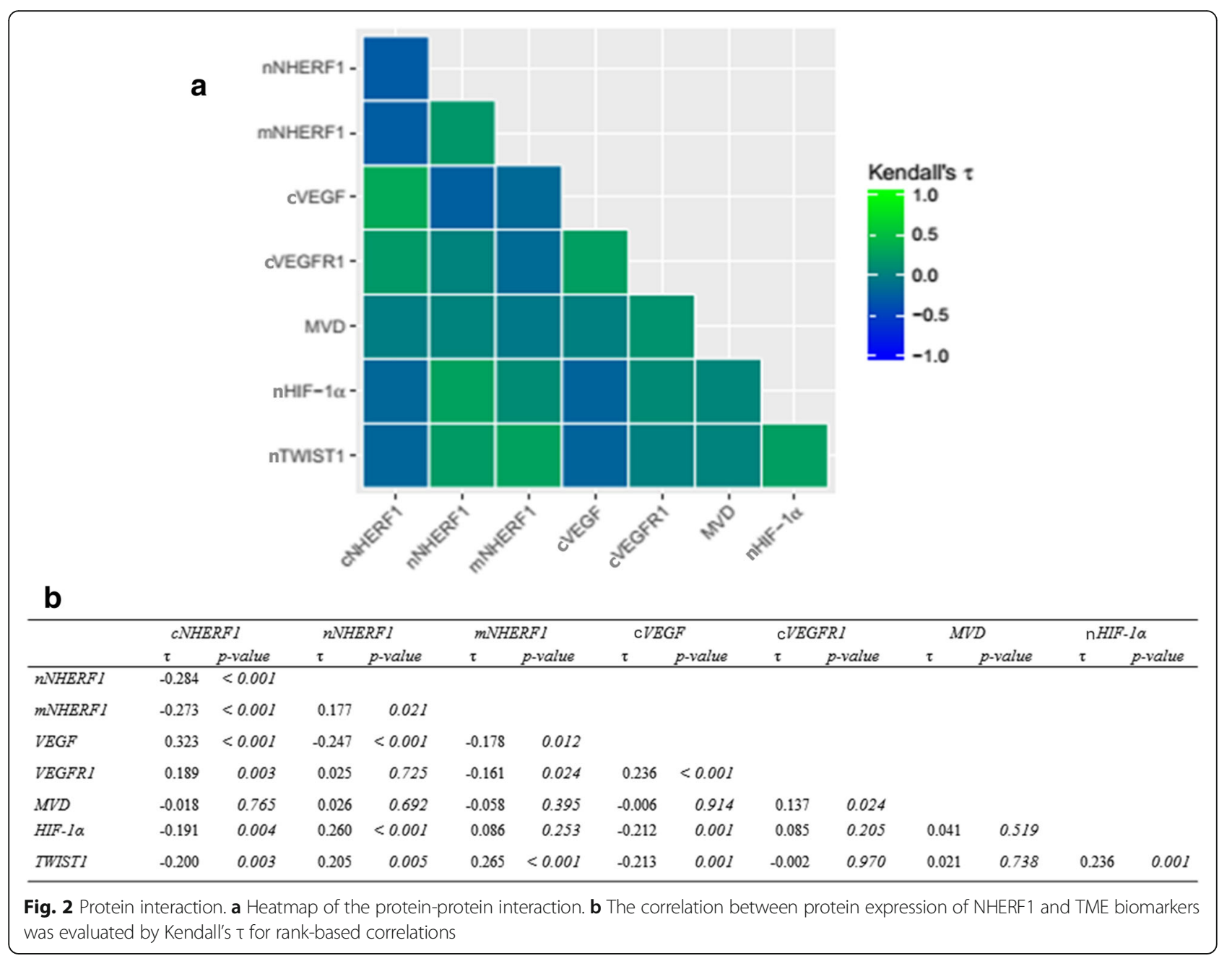

positive tumors $(p=0.021)$ while low nuclear and membranous NHERF1 was related to negative nHIF$1 \alpha$ expression $(p=0.001$ and $p=0.046)$ for $87.6 \%$ and $91 \%$ of cases, respectively. Kendall's $\tau$ test confirmed the presence of an inverse correlation between nHIF- $1 \alpha$ and CNHERF1 $(\tau=-0.191, p=0.004)$ and a positive correlation between nHIF- $1 \alpha$ and nNHERF1 $(\tau=0.260$, $p<0.001)$. Nuclear TWIST1 and cNHERF1 expression were detected in 130 tumors. Nuclear TWIST1 overexpression linked to a low cNHERF1 detection $(p=0.001)$ appeared in $71 \%$ of the cases while low expression of nNHERF1 and mNHERF1 was related to low nTWIST1 expression ( $p=0.008$ and $p=0.035)$ in $90.3 \%$ and $93.5 \%$ of tumors respectively. The data were also supported by Kendall's $\tau$ test, which emphasized a reverse behavior of nTWIST1 and cNHERF1 $(\tau=-0.200, p=0.003)$ and a positive relation between nTWIST1 and nuclear $(\tau=0$. 205, $p=0.005)$ and mNHERF1 $(\tau=0.265, p<0.001)$. No statistically significant association was found between MVD and NHERF1 for both dichotomized and continuous variables.
The analysis of the localization of VEGF and its receptor VEGFR1, by means of immunofluorescence studies, indicated that they co-localized with NHERF1, when both proteins were over-expressed within cytoplasmic and cytoplasmic and/or membranous compartments respectively, in invasive cellular clusters. Furthermore, our studies indicated that nHIF-1 $\alpha$ and nTWIST1 co-localized with NHERF1 too (Fig. 3a). It was interesting to observe that while an immunofluorescence assay showed a high MVD, a neo-vascular formation with a mixed labeling CD34/ NHERF1 was also noted (Fig. 3b).

Moreover, of the 183 total tumors, 145 could be assessed for both cVEGF and cVEGFR1, and $64.9 \%$ showed a positive relation between cVEGF and cVEGFR1 expression $(p=0.019)$. Of 141 tumor samples valuable for both cVEGF and nHIF- $1 \alpha, 62.2 \%$ of the tumor samples presented cVEGF negative expression and nHIF- $1 \alpha$ positive expression $(p=0.049)$. In addition, of the 128 tumors that could be assessed for both cVEGF and nTWIST1, 67.2\% of the samples presented cVEGF negative expression and nTWIST1 positive expression $(p=0.013)$. High MVD was 


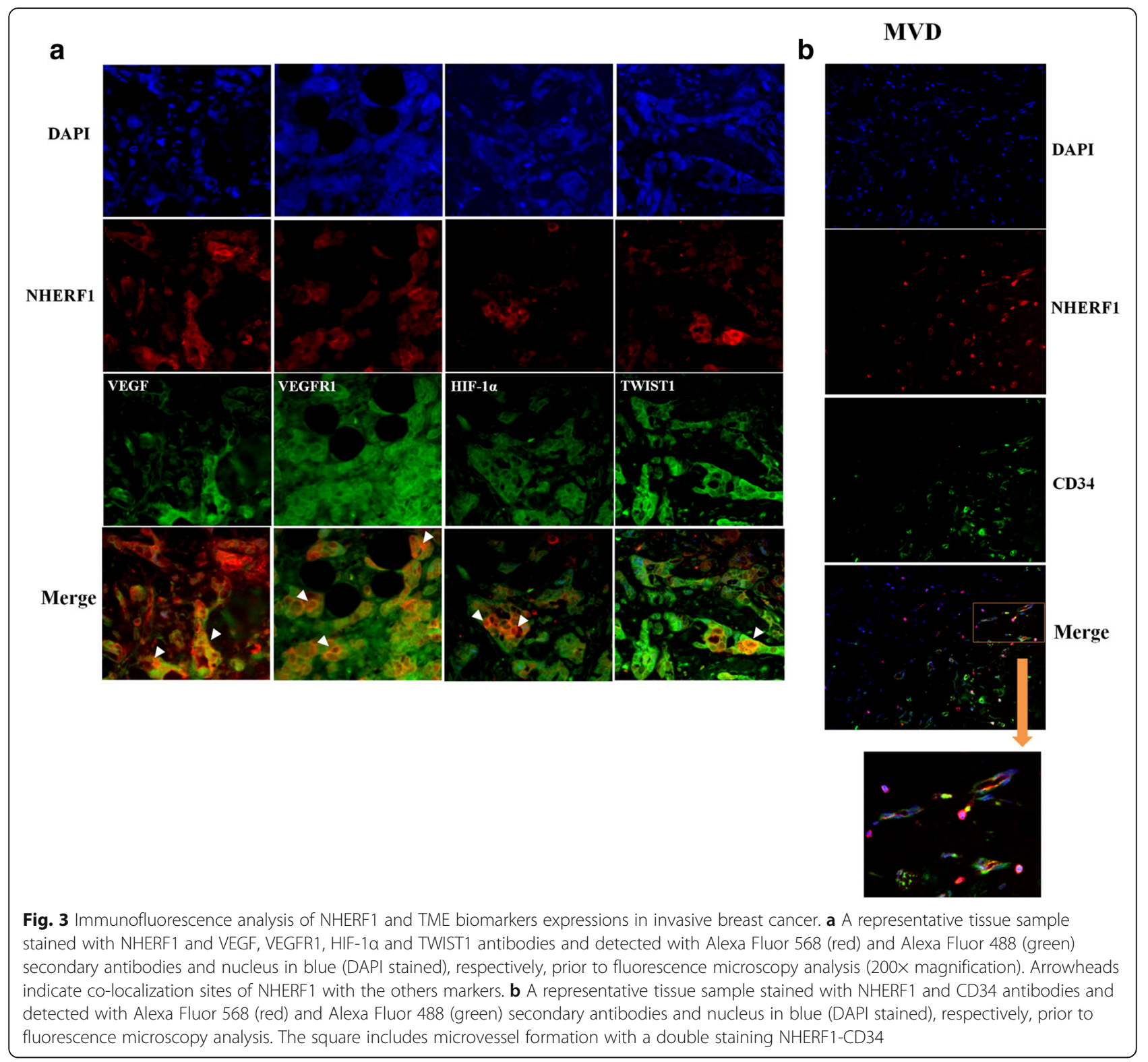

associated to cVEGFR1 positive expression $(p=0.013)$ and nHIF- $1 \alpha / n$ TWIST1 negative expressions were together related $(p=0.001)$ in $61.7 \%$ and $78.5 \%$ of the tumors, respectively (Table 3). Furthermore, Kendall's $\tau$ test also revealed a positive correlation between cVEGF and cVEGFR1 expression $(\tau=0.236, p<0.001)$, between cVEGFR1 expression and MVD ( $\mathrm{\tau}=0.137, p=0.024)$, and nHIF- $1 \alpha$ and nTWIST1 expression $(\tau=0.236, p=0.001)$. While, an inverse relation between cVEGF and nHIF- $1 \alpha(\tau=-0.212$, $p=0.001)$, cVEGF and nTWIST1 expression $(\tau=-0.213$, $p=0.001$ ), was observed (Fig. 2b).

\section{Expression of the proteins and patient outcome}

Univariate analyses were carried out for all the clinicopathological characteristics and the expression of cNHERF1,
nNHERF1, mNHERF1, cVEGF, cVEGFR1, MVD, nHIF-1 $\alpha$ and nTWIST1 proteins, as dichotomized variables. These were correlated to disease-free survival (DFS) and overallsurvival (OS) (Table 4). The subgroup of patients with negative expression of nTWIST1 had a shorter 5 -year DFS $(p<0.001)$; the same held true for continuous data $(p=0.022$, data not shown). Moreover, univariate analysis of clinicopathological characteristics in the entire cohort revealed that only MIB1 was significantly associated with worse DFS $(p=0.003)$.

According to the Cox proportional hazard regression model, multivariate analysis of the entire cohort (Table 5), identified nTWIST1 expression, in both categorical and continuous data $(\mathrm{HR}=0,14,95 \%$ confidence interval: CI $0.05-0.43, p=0.001$; and $\mathrm{HR}=0.92,95 \%$ CI $0.87-0.97$, 
Table 4 Univariate analysis with respect to DFS and OS in 118 patients with invasive breast cancer

\begin{tabular}{|c|c|c|c|c|c|c|c|c|}
\hline \multirow[b]{2}{*}{ Characteristics } & \multirow[b]{2}{*}{ No. pts. } & \multicolumn{4}{|l|}{ DFS } & \multicolumn{3}{|l|}{ OS } \\
\hline & & events & 5-year DFS $(95 \% \mathrm{Cl})^{a}$ & $\mathrm{HR}(95 \% \mathrm{Cl})^{b}$ & $\overline{p \text {-value }}$ & events & 5 -year OS $(95 \% \mathrm{Cl})^{a}$ & $p$-value \\
\hline Overall & 118 & 37 & $83(76,91)$ & & & 2 & $98(95,100)$ & \\
\hline \multicolumn{9}{|l|}{ Age } \\
\hline$\leq 49$ & 74 & 23 & $84(75,94)$ & 1.00 & 0.993 & 2 & $97(92,100)$ & 0.275 \\
\hline$>49$ & 44 & 14 & $81(69,95)$ & $1.00(0.511 .94)$ & & 0 & $100(100,100)$ & \\
\hline \multicolumn{9}{|l|}{ CNHERF1 } \\
\hline Negative (< 40\%) & 48 & 16 & $83(72,95)$ & 1.00 & 0.546 & 2 & $95(89,100)$ & 0.150 \\
\hline Positive ( $\geq 40 \%$ ) & 49 & 15 & $85(75,97)$ & $0.80(0.40,1.63)$ & & 0 & $100(100,100)$ & \\
\hline \multicolumn{9}{|l|}{ nNHERF1 } \\
\hline Negative (0\%) & 81 & 28 & $83(74,92)$ & 1.00 & 0.382 & 2 & $97(93,100)$ & 0.547 \\
\hline Positive (> 0\%) & 16 & 3 & $92(77,100)$ & $0.59(0.18,1.95)$ & & 0 & $100(100,100)$ & \\
\hline \multicolumn{9}{|l|}{ mNHERF1 } \\
\hline Negative (0\%) & 86 & 26 & $85(77,94)$ & 1.00 & 0.133 & 2 & $97(94,100)$ & 0.642 \\
\hline Positive (> 0\%) & 13 & 5 & $75(50,100)$ & $2.06(0.79,5.42)$ & & 0 & $100(100,100)$ & \\
\hline \multicolumn{9}{|l|}{ CVEGF } \\
\hline Negative (0-2) & 44 & 12 & $76(62,92)$ & 1.00 & 0.632 & 1 & $97(90,100)$ & 0.742 \\
\hline Positive (3-7) & 56 & 19 & $88(79,97)$ & $0.84(0.41,1.73)$ & & 1 & $98(95,100)$ & \\
\hline \multicolumn{9}{|l|}{ CVEGFR1 } \\
\hline Negative (0\%) & 50 & 16 & $83(73,95)$ & 1.00 & 0.427 & 1 & $97(92,100)$ & 0.930 \\
\hline Positive (> 0\%) & 56 & 15 & $87(78,97)$ & $0.75(0.37,1.52)$ & & 1 & $98(94,100)$ & \\
\hline \multicolumn{9}{|l|}{ MVD } \\
\hline Negative $\left(<15\right.$ microvessels $\left./ \mathrm{mm}^{2}\right)$ & 42 & 14 & $82(70,95)$ & 1.00 & 0.541 & 2 & $95(88,100)$ & 0.111 \\
\hline Positive ( $\geq 15$ microvessels/mm²) & 58 & 16 & $89(80,98)$ & $0.80(0.39,1.64)$ & & 0 & $100(100,100)$ & \\
\hline \multicolumn{9}{|l|}{$n H I F-1 a$} \\
\hline Negative (0\%) & 62 & 18 & $85(75,95)$ & 1.00 & 0.813 & 2 & $96(91,100)$ & 0.270 \\
\hline Positive (> 0\%) & 39 & 11 & $91(81,700)$ & $0.91(0.43,1.94)$ & & 0 & $100(100,100)$ & \\
\hline \multicolumn{9}{|l|}{ nTWIST1 } \\
\hline Negative (4\%) & 46 & 20 & $77(65,91)$ & 1.00 & $<0.001$ & 1 & $97(92,100)$ & 0.304 \\
\hline Positive (> 4\%) & 47 & 8 & $92(85,100)$ & $0.25(0.10,0.58)$ & & 0 & $100(100,100)$ & \\
\hline \multicolumn{9}{|l|}{ Hystological type } \\
\hline CDI & 105 & 32 & $84(77,92)$ & 1.00 & 0.476 & 2 & $98(95,100)$ & 0.894 \\
\hline CLI & 9 & 4 & $59(92,100)$ & $1.83(0.64,5.18)$ & & 0 & $100(100,100)$ & \\
\hline Other & 4 & 1 & $100(100,100)$ & $0.72(0.10,5.25)$ & & 0 & $100(100,100)$ & \\
\hline \multicolumn{9}{|l|}{ Hystological grade } \\
\hline G1 & 14 & 4 & $91(75,100)$ & 1.00 & 0.874 & 0 & $100(100,100)$ & 0.240 \\
\hline G2 & 53 & 17 & $83(73,94)$ & $1.30(0.44,3.88)$ & & 0 & $100(100,100)$ & \\
\hline G3 & 51 & 16 & $81(70,94)$ & $0.32(0.44,3.97)$ & & 2 & $95(89,100)$ & \\
\hline \multicolumn{9}{|l|}{ Tumor size (cm) } \\
\hline$\leq 2 \mathrm{~cm}$ & 44 & 14 & $93(85,100)$ & 1.00 & 0.675 & 0 & $100(100,100)$ & 0.242 \\
\hline$>2 \mathrm{~cm}$ & 72 & 21 & $80(69,90)$ & $1.16(0.59,2.28)$ & & 2 & $97(92,100)$ & \\
\hline \multicolumn{9}{|l|}{ Receptor status } \\
\hline ER-Negative ( $\leq 10 \%)$ & 38 & 10 & $80(68,96)$ & 1.00 & 0.675 & 2 & $94(85,100)$ & 0.033 \\
\hline ER-Positive (> 10\%) & 80 & 27 & $84(76,93)$ & $1.16(0.56,2.42)$ & & 0 & $100(100,100)$ & \\
\hline PgR-Negative ( $\leq 10 \%)$ & 47 & 18 & $76(64,90)$ & 1.00 & 0.193 & 2 & $95(89,100)$ & 0.092 \\
\hline
\end{tabular}


Table 4 Univariate analysis with respect to DFS and OS in 118 patients with invasive breast cancer (Continued)

\begin{tabular}{|c|c|c|c|c|c|c|c|c|}
\hline \multirow[b]{2}{*}{ Characteristics } & \multirow[b]{2}{*}{ No. pts. } & \multicolumn{4}{|l|}{ DFS } & \multicolumn{3}{|l|}{ OS } \\
\hline & & events & 5-year DFS $(95 \% \mathrm{Cl})^{a}$ & $\operatorname{HR}(95 \% \mathrm{Cl})^{b}$ & $\overline{p \text {-value }}$ & events & 5 -year OS $(95 \% \mathrm{Cl})^{a}$ & $p$-value \\
\hline PgR-Positive (> 10\%) & 71 & 19 & $88(80,97)$ & $0.65(0.34,1.25)$ & & 0 & $100(100,100)$ & \\
\hline \multicolumn{9}{|l|}{ MIB-1 } \\
\hline Negative $(\leq 20 \%)$ & 47 & 7 & $87(77,98)$ & 1.00 & 0.003 & 1 & $97(92,100)$ & 0.773 \\
\hline Positive (> 20\%) & 71 & 30 & $80(71,91)$ & $3.20(1.40,7.30)$ & & 1 & $98(96,100)$ & \\
\hline \multicolumn{9}{|l|}{ HER2/neu } \\
\hline Negative $(0 /+1)$ & 59 & 19 & $88(79,98)$ & 1.00 & 0.780 & 0 & $100(100,100)$ & 0.098 \\
\hline Positive $(+3)$ & 45 & 13 & $75(61,90)$ & $1.11(0.55,2.24)$ & & 2 & $94(87,100)$ & \\
\hline
\end{tabular}

${ }^{a}$ five-year disease-free survival (DFS) or overall survival (OS) based on Kaplan-Meier method, ${ }^{b}$ hazard-ratio (HR) computed using the Cox proportional hazard regression model for the DFS (for OS cannot be computed due to the low number of events), ${ }^{c} p$-value of the log-rank test for the equality of probability of an event (relapse for DFS or death for OS)

$p=0.003$, respectively) as significant for DFS. Moreover, for continuous data $(\mathrm{HR}=1.09,95 \% \mathrm{CI} 1.02-1.17, p=0$. 011), mNHERF1 expression was a significant indicator of DFS (Table 5) as well. HR for OS was not computed due to the low number of events.

The relationship among the different analyzed biomarkers expression and BCs survival was then investigated. Kaplan-Meier curves revealed that patients with nTWIST1expression had a worse DFS than of patients with nTWIST1+ expression (5-years $77 \%$ vs $92 \%, p<0.001$ ) (Fig. 4a).

When nTWIST1 and mNHERF1 expressions were considered, Kaplan-Meier curves showed that patients with nTWIST1-/mNHERF1+ immunophenotype had a better DFS respect to patients with nTWIST1+/mNHERF1$(p<0.001)$ (Fig. 4b). Analyzing patients with nTWIST1-/ mNHERF1- vs nTWIST1+/mNHERF1+ expression, no statistically significant results were found ( $p=0.510$ ), (Fig. 4c).

In addition, the subgroup of patients nTWIST1-/ cNHERF1- showed a higher DFS with respect to the subgroup nTWIST1+/cNHERF1+ $(p=0.004)$. When

Table 5 Multivariate analysis with respect to DFS and OS in invasive breast cancer

\begin{tabular}{|c|c|c|c|c|}
\hline \multirow[b]{2}{*}{ Characteristics } & \multicolumn{2}{|l|}{ categorical } & \multicolumn{2}{|l|}{ continuous } \\
\hline & $\overline{\mathrm{HR}(95 \% \mathrm{Cl})^{a}}$ & $\overline{p \text {-value }}{ }^{a}$ & $\overline{\mathrm{HR}(95 \% \mathrm{Cl})^{a}}$ & $p$-value \\
\hline CNHERF1 & $0.63(0.25,1.60)$ & 0.337 & $0.99(0.96,1.02)$ & 0.416 \\
\hline nNHERF1 & $0.60(0.07,5.167)$ & 0.640 & $0.89(0.70,1.12)$ & 0.319 \\
\hline mNHERF1 & $3.71(0.98,13.99)$ & 0.053 & $1.09(1.02,1.17)$ & 0.011 \\
\hline CVEGF & $0.63(0.25,1.61)$ & 0.333 & $0.99(0.97,1.01)$ & 0.249 \\
\hline CVEGFR1 & $0.64(0.28,1.48)$ & 0.297 & $1.00(0.98,1.02)$ & 0.826 \\
\hline MVD & $0.54(0.23,1.29)$ & 0.165 & $0.97(0.91,1.04)$ & 0.442 \\
\hline$n H I F-1 a$ & $0.69(0.24,2.02)$ & 0.504 & $0.99(0.95,1.03)$ & 0.520 \\
\hline nTWIST1 & $0.14(0.05,0.43)$ & 0.001 & $0.92(0.87,0.97)$ & 0.003 \\
\hline
\end{tabular}

${ }^{a}$ hazard-ratio (HR) and $p$-value for DFS computed using the multivariate Cox proportional hazard regression model with categorical and continuous variables; HR for OS cannot be computed due to the low number of events
nTWIST1-/cNHERF1+ vs nTWIST1+/cNHERF1phenotype were compared, the first group showed a worse DFS ( $p=0.037)$ (Fig. 4d, e). Kaplan-Meier curves, computed for the other proteins analyzed, revealed that there were no statistically significant results comparing the DFS of the group of patients with cVEGF-/cNHERF1- vs $c \mathrm{VEGF}+/ \mathrm{cNHERF} 1+$ expression $(p=0.430)$ (Fig. 4f), and with cVEGFR1-/cNHERF1- vs cVEGFR1+/cNHERF1+ expression $(p=0.180)$ (Fig. $4 \mathrm{~g})$ and nHIF-1 $\alpha$-/cNHERF1+ vs nHIF- $1 \alpha+/$ cNHERF1- expression $(p=0.950)$ (Fig. 4h). There were no significant correlations for protein levels and OS.

\section{Discussion}

The study regarding cancer context has become of great significance because of the therapeutic implications linked to it. In recent years, the complexity of TME has been evidenced enough for it to be considered as a real system in an ever-changing state.

In this study, a panel of factors involved in TME changes such as VEGF, VEGFR1, HIF-1a, TWIST1 and $\mathrm{MVD}$, that have been related to $\mathrm{BC}$ progression, aggressiveness and invasion was analyzed [6, 12, 28-30]. Further, so as to better characterize this microenvironment, their association with the scaffolding protein NHERF1 and with survival was observed, allowing to identify a possible subgroup of patients that could benefit from a specific therapy.

Mainly, in this BC cohort there was evidence of an increase of cNHERF1 expression with a loss of mNHERF1 detection and a small nNHERF1 presence, in conformity with a more aggressive behavior. Our group had already shown that the switch from apical membranous to cytoplasmic expression was linked to breast carcinogenesis, with a high cNHERF1 expression, present in more aggressive tumors [23]. In addition, the loss of nNHERF1 expression had also been associated with reduced survival, indicating it as an independent prognostic marker in BC [25]. An increase of TME analyzed markers had 


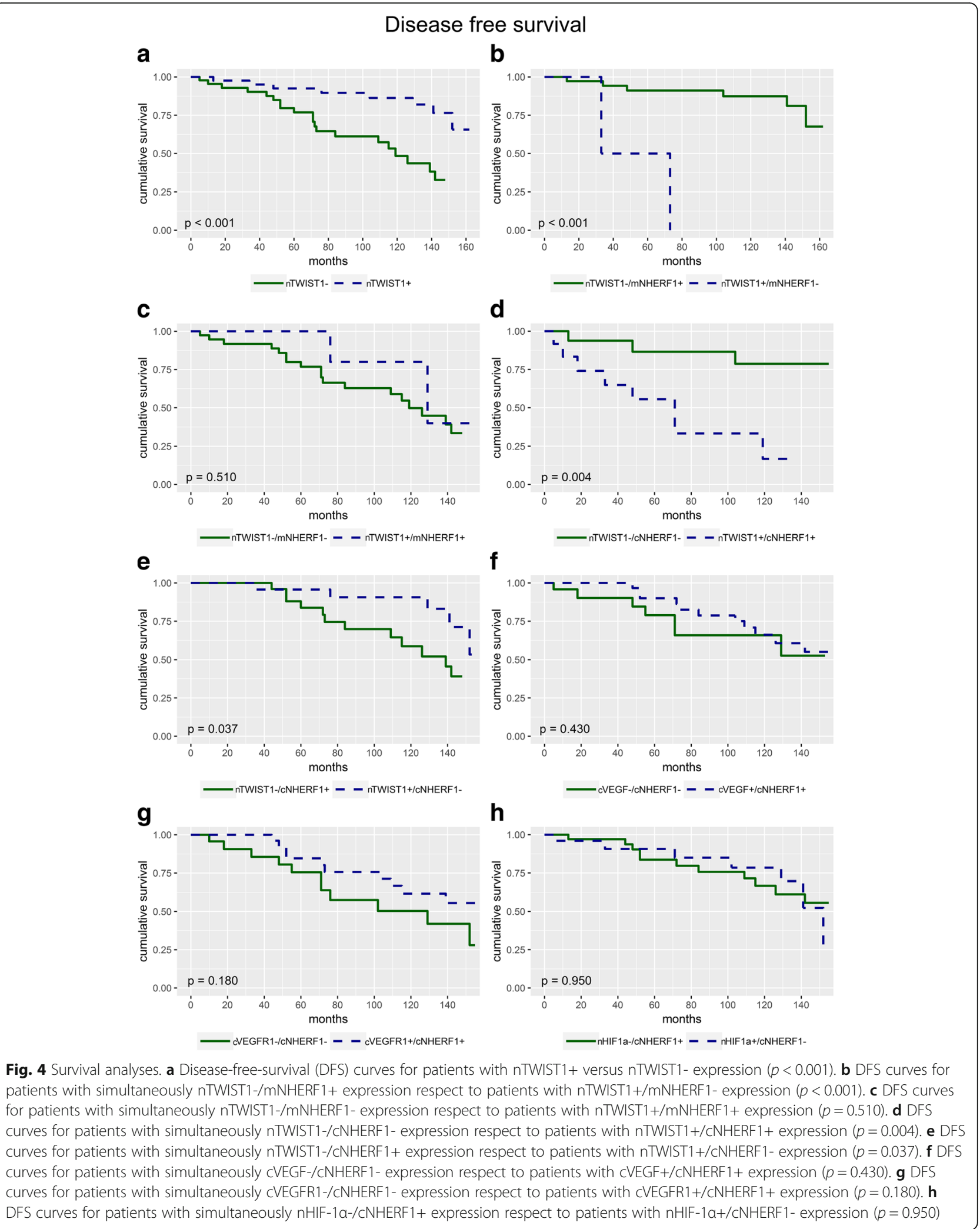


been reported in these tumors, to confirm their aggressiveness. Moreover, it was shown that cNHERF1 overexpression was related to some aggressive clinical parameters, as demonstrated by association with extensive tumor size and high proliferative activity. Membranous and nuclear NHERF1 expression reaffirmed their protective role, being related to low proliferative activity and smaller tumors. Moreover, our results supported that cVEGF might have a potential additional predictive value, as demonstrated in this population, including younger patients with larger tumors, extensive lymph-node metastases and high grade and proliferative activity. Its receptor cVEGFR1 over-expression was associated to an invasive phenotype as well. VEGF and VEGFR1 have been indicated as strong biomarker indicators to select patients who could benefit from anti-angiogenic therapy [31]. Despite this is an innovative approach, therapeutical failures projected for novel solutions to improve and overcome actual limits [32]. It was e also demonstrated, in this study, that CNHERF1 overexpression was positively related with cVEGF expression. Interestingly, a significant direct association between increased levels of cNHERF1 and cVEGFR1 was also noticed. At the same time, membranous and nuclear NHERF1 reconfirmed their defensive role, being negatively related to cVEGF expression. VEGF is well known as the principal modulator in the process of cancer growth, invasion and metastasis, through new microvessel formation and lymphovascular invasion [33]. Previous studies carried out by our équipe had already reported a NHERF1/VEGFR1 relationship with poor clinical outcome in invasive $\mathrm{BCs}$, indicating a possible interaction between the two proteins [19]. VEGF and VEGFR1 high expression is part of highly hypoxic and aggressive environments, involving high HIF-1 $\alpha$ and TWIST1 levels of expression too. Several studies have identified a relation to poorer prognosis/therapy response in the altered expression of HIF-1 $\alpha$ and TWIST1 [34-36]. Moreover, the hypoxic environment in $\mathrm{BCs}$ is one of the key regulators of the network of the biological elements of matrix, involved in metastasis process and it affects both the early and late stages of metastasis [37]. The dissemination process induced by hypoxia is supplied by paracrine and autocrine stimuli and it is interconnected with EMT program and characterized by high expression of different proteins $[38,39]$. The results in this study indicated a direct relation between nHIF- $1 \alpha$ and nTWIST1 expression, but a controversial action was present, being both nHIF-1 $\alpha$ and nTWIST1 loss interrelated to more aggressive phenotype. Furthermore, an inverse relation was found between cNHERF1 and nHIF- $1 \alpha$ and cNHERF1 and nTWIST1 expression, even if a trend consistent with CNHERF1 positive expression was expected, being it correlated to $\mathrm{BCs}$ more aggressive phenotype [18, 23, 25]. Additionally, atypically, high nHIF- $1 \alpha$ and nTWIST1 expression was present in tumors with low cVEGF expression. Different factors could have contributed to these results, especially knowing that TME is characterized by continuous changing. A possible reason for these discrepancies could be the heterogeneity of BC. Cancer is inherently a heterogeneous disease and so heterogeneity has become its main feature. Clinical and histo-pathological parameters, biomarkers and genetic heterogeneity can be affected by the different characteristics of a single patient (age, menopausal state, general health status etc), but also by epigenetic changes (histone modification, DNA methylation etc) $[40,41]$ and metabolic reprogramming [42]. Expression of biomarkers can be highly variable within an individual tumor, causing interpretation problems and discordant results.

Regarding nTWIST1 negative expression, it was observed that most of these tumors presented more aggressive clinic-pathological characteristics (high proliferative activity, positive lymph node status, large tumor size, high histological grade and HER2-postive status), despite the fact that there was not a significant statistical evidence for any of these.

Evidence of all this scenario was the presence of new vessels formation, detected by immunohistochemistry and immunofluorescence assay. MVD showed only a direct association with cVEGFR1 over-expression, while no other significant relation has been found. Remarkably, in the tumor area, a neo-vascular formation with a "mosaic" structure was observed with a double staining of CD34 and NHERF1, which could indicate a probable tumor cell recruitment in neo blood vessels formation with endothelial cells. This is a well-known mechanism, which in addition to sprouting angiogenesis, has been recognized to contribute to tumor vascularization. In fact, new blood vessels can be due to vasculogenic mimicry formation, that comprise not only vessels formed by endothelial cells, but also tubules formed by malignant cancer cells [43-45]. NHERF1 involvement in neovascularization phenomena has already been reported [46, 47], but this is the first evidence of a possible direct participation of tumor cells NHERF1 positive to new-vessels formation.

In a subgroup of patients with long-term follow-up, multivariate analysis showed that loss nTWIST1 expression was related to a decrease of DFS. Other authors had reported a worse DFS linked to TWIST1 negative expression, suggesting that its deregulation was involved in poor patient outcome [48, 49]. The Kaplan Meyer curves were used to evaluate the relationship between the biomarkers. Interestingly, when nTWIST1-/mNHERF1+ phenotype were considered, it was discovered that these patients showed a better DFS with respect to nTWIST1 +/mNHERF1- phenotype. In addition, data showed a worse outcome in patients nTWIST1-/mNHERF1- vs nTWIST1+/mNHERF1+, despite it being not statistically significant. These findings confirmed the oncosuppressor 
role of NHERF1 expression, when it was localized at the plasma membrane [50-52], suggesting that MNHERF1 expression might interfere and influence clinical outcome.

Thus, the analyses of nTWIST1+/cNHERF1+ patients selected a phenotype with a worse DFS compared to nTWIST1-/cNHERF1-, as well as the nTWIST1-/ cNHERF1+ with respect to nTWIST1+/cNHERF1-, corroborating the pivotal role of CNHERF1 in cancer progression, repeatedly stated by previous researches [18, 23-25].

\section{Conclusions}

In conclusion, the study reported a closer interaction of cNHERF1 with cVEGF, cVEGFR1 and nTWIST1, that denoted an active participation of tumor microenvironment to cancer aggressiveness. From this point of view, it is very interesting to consider the mimicry involving NHERF $1^{+}$ tumor cells in neo-microvessels formation, and this opens a new stimulating scenario for further NHERF1 studies. Moreover, the identification of nTWIST1-/mNHERF1+ phenotype as a subgroup with an increased DFS and nTWIST1+/cNHERF1+, nTWIST1-/cNHERF1+ phenotypes as patient subgroups with poor outcome, reinforced both the tumor suppressor role of mNHERF1 and oncogenic activity of cNHERF1. Furthermore, these date suggest the potential prognostic role of NHERF1. Finally, we believe that our intriguingly results warrant additional studies aimed to evaluate tumor microenvironment regulation in combination with NHERF1 as a possible targeted-oriented therapeutic approach.

\section{Additional files}

Additional file 1: Table S1. Dilution, source, staining of antibodies and cut off used. (DOC $44 \mathrm{~kb})$

Additional file 2: Table S2. Expression frequency of biomarkers. (DOC $36 \mathrm{~kb}$ )

\section{Abbreviations \\ BC: breast cancer; Cl: confidence interval; CNHERF1: cytoplasmic NHERF1; CVEGF: cytoplasmic VEGF; cVEGFR1: cytoplasmic VEGFR1; DFS: Disease-free survival; EMT: epithelial to mesenchimal transition; ER: estrogen receptor; HER2: human epidermal growth factor receptor 2; HIF-1a: hypoxia inducible factor-1 alpha; HIS: immunohistochemical score; HR: hazard ratio; \\ IHC: immunohistochemistry; mNHERF1: membranous NHERF1; MVD: microvesse density; NHERF1: $\mathrm{Na}^{+} / \mathrm{H}^{+}$exchanger regulatory factor 1 ; $\mathrm{nHIF-1a:} \mathrm{nuclear} \mathrm{HIF-1a}$; nNHERF1: nuclear NHERF1; nTWIST1: nuclear TWIST1; OS: overall survival; PgR: progesterone receptor; TMA: tissue microarray; TME: tissue microenvironment; VEGF: vascular endothelial growth factor; VEGFR1: vascular endothelial growth factor receptor 1}

\section{Acknowledgements}

The authors would like to thank Francesco Fanelli and Valeria Albenzio for technical assistance. and Bianca Tino for language revision and editing.

\section{Funding}

This work was supported by funding from the Italian Ministry of Health "Ricerca Corrente 2017".

\section{Availability of data and materials}

All data generated or analyzed during this study were included in this published article.

\section{Authors' contributions}

CS planned the study, performed the experiments and participated in the drafting of the manuscript. AV and ES performed the statistical analyses. MC, IC and NS helped to revise the manuscript. OP and MIP collected the samples and performed the diagnosis. FG provided the clinical data. AM is responsible for the critical revision of the article and scientific supervision. All authors read and approved the final manuscript.

\section{Ethics approval and consent to participate}

This study was approved by the Ethic Committee of Istituto Tumori "Giovanni Paolo II" with the reference number 234/CE 13-11-2017. Before undergoing routine surgery, all patients signed an informed consent form authorizing the Institute to utilize their removed biological tissue for research purposes according to ethical standards.

\section{Competing interests}

The authors declare that they have no competing interests.

\section{Publisher's Note}

Springer Nature remains neutral with regard to jurisdictional claims in published maps and institutional affiliations.

\section{Author details}

${ }^{1}$ Functional Biomorphology Laboratory, IRCCS-Istituto Tumori "Giovanni Paolo II", 70124 Bari, Italy. ${ }^{2}$ Unit of Biostatistics and Clinical Trials, Istituto Scientifico Romagnolo per lo Studio e la Cura dei Tumori (IRST)-IRCCS, 47014 Meldola, FC, Italy. "Pathology Department, IRCCS-Istituto Tumori "Giovanni Paololl", 70124 Bari, Italy. ${ }^{4}$ Medical Oncology Unit, IRCCS-Istituto Tumori "Giovanni Paolo II", 70124 Bari, Italy. ${ }^{5}$ Scientific Direction, IRCCS-Istituto Tumori "Giovanni Paolo II", 70124 Bari, Italy.

Received: 12 March 2018 Accepted: 19 April 2018

Published online: 02 May 2018

\section{References}

1. Quail DF, Joyce JA. Microenvironmental regulation of tumor progression and metastasis. Nat Med. 2013;19:1423-37.

2. Spill F, Reynolds DS, Kamm RD, Zaman MH. Impact of the physical microenvironment on tumor progression and metastasis. Curr Opin Biotechnol. 2016;40:41-8.

3. Ghoncheh M, Pournamdar Z, Salehiniya H. Incidence and Mortality and Epidemiology of Breast Cancer in the World. Asian Pac J Cancer Prev. 2016; 17:43-6.

4. Alizadeh AA, Aranda V, Bardelli A, Blanpain C, Bock C, Borowski C, et al. Toward understanding and exploiting tumor heterogeneity. Nat Med. 2015;21:846-53.

5. Andreuzzi E, Colladel R, Pellicani R, Tarticchio G, Cannizzaro R, Spessotto $P$, et al. The angiostatic molecule Multimerin 2 is processed by MMP-9 to allow sprouting angiogenesis. Matrix Biol. 2017;64:40-53.

6. Saponaro C, Malfettone A, Ranieri G, Danza K, Simone G, Paradiso A, et al. VEGF, HIF-1 a expression and MVD as an angiogenic network in familial breast cancer. PLoS One. 2013:8:e53070.

7. De Francesco EM, Pellegrino M, Santolla MF, Lappano R, Ricchio E, Abonante $\mathrm{S}$, et al. GPER mediates activation of HIF1a/NEGF signaling by estrogens. Cancer Res. 2014;74:4053-64

8. Kaufhold S, Bonavida B. Central role of Snail1 in the regulation of EMT and resistance in cancer: a target for therapeutic intervention. J Exp Clin Cancer Res. 2014;33:62.

9. Zhu QQ, Ma C, Wang Q, Song Y, Lv T. The role of TWIST1 in epithelialmesenchymal transition and cancers. Tumour Biol. 2016;37:185-97.

10. Sun X, Cui M, Zhang A, Tong L, Wang K, Li K, et al. MiR-548c impairs migration and invasion of endometrial and ovarian cancer cells via downregulation of Twist. J Exp Clin Cancer Res. 2016;35:10.

11. Vesuna F, Bergman Y, Raman V. Genomic pathways modulated by Twist in breast cancer. BMC Cancer. 2017;17:52

12. Schirosi L, De Summa S, Tommasi S, Paradiso A, Gasparini G, Popescu O, et al. VEGF and TWIST1 in a 16-biomarker immunoprofile useful for prognosis of breast cancer patients. Int J Cancer. 2017;141:1901-11. 
13. Sossey-Alaoui K, Pluskota E, Davuluri G, Bialkowska K, Das M, Szpak D, et al. Kindlin-3 enhances breast cancer progression and metastasis by activating Twist-mediated angiogenesis. FASEB J. 2014;28:2260-71.

14. Dunn HA, Ferguson SS. PDZ Protein Regulation of G Protein-Coupled Receptor Trafficking and Signaling Pathways. Mol Pharmacol. 2015;88:624-39.

15. Mangia A, Scarpi E, Partipilo G, Schirosi L, Opinto G, Giotta F, et al. NHERF1 together with PARP1 and BRCA1 expression as a new potential biomarker to stratify breast cancer patients. Oncotarget. 2017;8:65730-42.

16. Georgescu MM, Mobley BC, Orr BA, Shang P, Lehman NL, Zhu X, et al. NHERF1/EBP50 and NF2 as diagnostic markers for choroid plexus tumors. Acta Neuropathol Commun. 2016;4:55.

17. Mangia A, Caldarola L, Dell'Endice S, Scarpi E, Saragoni L, Monti M, et al. The potential predictive role of nuclear NHERF1 expression in advanced gastric cancer patients treated with epirubicin/oxaliplatin/capecitabine first line chemotherapy. Cancer Biol Ther. 2015;16:1140-7.

18. Cardone RA, Bellizzi A, Busco G, Weinman EJ, Dell'Aquila ME, Casavola V, et al. The NHERF1 PDZ2 domain regulates PKA-RhoA-p38-mediated NHE1 activation and invasion in breast tumor cells. Mol Biol Cell. 2007;18:1768-80.

19. Malfettone A, Saponaro C, Paradiso A, Simone G, Mangia A. Peritumoral vascular invasion and NHERF1 expression define an immunophenotype of grade 2 invasive breast cancer associated with poor prognosis. BMC Cancer. 2012;12:106.

20. Malfettone A, Silvestris N, Paradiso A, Mattioli E, Simone G, Mangia A. Overexpression of nuclear NHERF1 in advanced colorectal cancer: association with hypoxic microenvironment and tumor invasive phenotype. Exp Mol Pathol. 2012;92:296-303.

21. Gu Y, Yu H, Hao C, Martin TA, Hargest R, He J, et al. NHERF1 regulates the progression of colorectal cancer through the interplay with VEGFR2 pathway. Oncotarget. 2017;8:7753-65.

22. Wolff AC, Hammond ME, Schwartz JN, Hagerty KL, Allred DC, Cote RJ, et al. American Society of Clinical Oncology/College of American Pathologists guideline recommendations for human epidermal growth factor receptor 2 testing in breast cancer. J Clin Oncol. 2007;25:118-45.

23. Mangia A, Chiriatti A, Bellizzi A, Malfettone A, Stea B, Zito FA, et al. Biological role of NHERF1 protein expression in breast cancer. Histopathology. 2009;55:600-8.

24. Schirosi L, De Summa S, Tommasi S, Paradiso A, Sambiasi D, Popescu O, et al. Immunoprofile from tissue microarrays to stratify familial breast cancer patients. Oncotarget. 2015;6:27865-79.

25. Paradiso A, Scarpi E, Malfettone A, Addati T, Giotta F, Simone G, et al. Nuclear NHERF1 expression as a prognostic marker in breast cancer. Cell Death Dis. 2013;4:e904.

26. Mangia A, Malfettone A, Saponaro C, Tommasi S, Simone G, Paradiso A. Human epidermal growth factor receptor $2, \mathrm{Na}+/ \mathrm{H}+$ exchanger regulatory factor 1, and breast cancer susceptibility gene-1 as new biomarkers for familial breast cancers. Hum Pathol. 2011;42:1589-95.

27. R Core Team. R: A language and environment for statistical computing. Vienna: R Foundation for Statistical Computing; 2017. https://www.R-project. org/.

28. Zhang L, Wang H, Li C, Zhao Y, Wu L, Du X, et al. VEGF-A/Neuropilin 1 pathway confers Cancer Stemness via activating Wnt/B-catenin Axis in breast Cancer cells. Cell Physiol Biochem. 2017;44:1251-62.

29. Weddell JC, Chen S, Imoukhuede PI. EGFR1 promotes cell migration and proliferation through PLCY and PI3K pathways. NPJ Syst Biol Appl. 2017;4:1.

30. Liang G, Liu Z, Tan L, Su AN, Jiang WG, Gong C. HIF1a-associated circDENND4C promotes proliferation of breast Cancer cells in hypoxic environment. Anticancer Res. 2017;37:4337-43.

31. Lambrechts D, Lenz HJ, de Haas S, Carmeliet P, Scherer SJ. Markers of response for the antiangiogenic agent bevacizumab. J Clin Oncol. 2013;31:1219-30.

32. Xu Y, Li Q, Li XY, Yang QY, Xu WW, Liu GL. Short-term anti-vascular endothelial growth factor treatment elicits vasculogenic mimicry formation of tumors to accelerate metastasis. J Exp Clin Cancer Res. 2012;31:16.

33. Sa-Nguanraksa D, Chuangsuwanich T, Pongpruttipan T, O-Charoenrat P. High vascular endothelial growth factor gene expression predicts poor outcome in patients with non-luminal A breast cancer. Mol Clin Oncol. 2015;3:1103-8

34. Jin MS, Lee $H$, Park IA, Chung YR, Im SA, Lee KH, et al. Overexpression of HIF1a and CAXI predicts poor outcome in early-stage triple negative breast cancer. Virchows Arch. 2016:469:183-90.

35. Semenza GL. Targeting HIF-1 for cancer therapy. Nat Rev Cancer. 2003;3:721-32.
36. Grzegrzolka J, Wojtyra P, Biala M, Piotrowska A, Gomulkiewicz A, Rys J, et al. Correlation between expression of Twist and Podoplanin in ductal breast carcinoma. Anticancer Res. 2017;37:5485-93.

37. Rankin EB, Giaccia AJ. Hypoxic control of metastasis. Science. 2016;352:175-80.

38. Ye X, Weinberg RA. Epithelial-mesenchymal plasticity: a central regulator of cancer progression. Trends Cell Biol. 2015;25:675-86.

39. Tiwari N, Gheldof A, Tatari M, Christofori G. EMT as the ultimate survival mechanism of cancer cells. Semin Cancer Biol. 2012;22:194-207.

40. Beca F, Polyak K. Intratumor Heterogeneity in Breast Cancer. Adv Exp Med Biol. 2016;882:169-89.

41. Turashvili G, Brogi E. Tumor Heterogeneity in Breast Cancer. Front Med (Lausanne). 2017:4:227.

42. Yoshida GJ. Metabolic reprogramming: the emerging concept and associated therapeutic strategies. J Exp Clin Cancer Res. 2015;34:111.

43. Zhang S, Zhang D, Sun B. Vasculogenic mimicry: current status and future prospects. Cancer Lett. 2007;254:157-64.

44. Zang M, Zhang Y, Zhang B, Hu L, Li J, Fan Z, et al. CEACAM6 promotes tumor angiogenesis and vasculogenic mimicry in gastric cancer via FAK signaling. Biochim Biophys Acta. 2015;1852:1020-8.

45. Kaessmeyer S, Bhoola K, Baltic S, Thompson P, Plendl J. Lung cancer neovascularisation: Cellular and molecular interaction between endothelial and lung cancer cells. Immunobiology. 2014;219:308-14.

46. Hou Y, Wu Y, Farooq SM, Guan X, Wang S, Liu Y, et al. A critical role of CXCR2 PDZ-mediated interactions in endothelial progenitor cell homing and angiogenesis. Stem Cell Res. 2015;14:133-43.

47. Chen $P$, Wang $Y$, Yang L, Li C, Wang $Y$, Xie L, et al. Novel bioactivity of NHERF1 in corneal neovascularization. Graefes Arch Clin Exp Ophthalmol. 2012;250:1615-25.

48. Montserrat N, Gallardo A, Escuin D, Catasus L, Prat J, Gutiérrez-Avignó FJ, et al. Repression of E-cadherin by SNAIL, ZEB1, and TWIST in invasive ductal carcinomas of the breast: a cooperative effort? Hum Pathol. 2011:42:103-10.

49. Cates JM, Byrd RH, Fohn LE, Tatsas AD, Washington MK, Black CC. Epithelialmesenchymal transition markers in pancreatic ductal adenocarcinoma. Pancreas. 2009;38:e1-6.

50. Molina JR, Agarwal NK, Morales FC, Hayashi Y, Aldape KD, Cote G, et al. PTEN, NHERF1 and PHLPP form a tumor suppressor network that is disabled in glioblastoma. Oncogene. 2012;31:1264-74.

51. Pan $Y$, Wang $L$, Dai JL. Suppression of breast cancer cell growth by $\mathrm{Na}+/ \mathrm{H}+$ exchanger regulatory factor 1 (NHERF1). Breast Cancer Res. 2006;8:R63.

52. Kreimann EL, Morales FC, de Orbeta-Cruz J, Takahashi Y, Adams H, Liu TJ, et al. Cortical stabilization of beta-catenin contributes to NHERF1/ EBP50 tumor suppressor function. Oncogene. 2007;26:5290-9.

\section{Ready to submit your research? Choose BMC and benefit from:}

- fast, convenient online submission

- thorough peer review by experienced researchers in your field

- rapid publication on acceptance

- support for research data, including large and complex data types

- gold Open Access which fosters wider collaboration and increased citations

- maximum visibility for your research: over $100 \mathrm{M}$ website views per year

At BMC, research is always in progress.

Learn more biomedcentral.com/submissions 